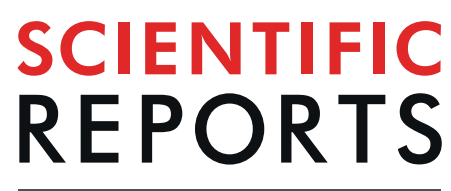

natureresearch

\title{
OPEN
}

\section{L-type voltage-gated calcium channel regulation of in vitro human cortical neuronal networks}

Received: 30 November 2018

Accepted: 2 September 2019

Published online: 25 September 2019
William Plumbly ${ }^{1}$, Nick Brandon ${ }^{2}{ }^{2}$, Tarek Z. Deeb $\mathbb{D}^{3}{ }^{3}$ Jeremy Hall ${ }^{1}{ }^{1} \&$ Adrian J. Harwood $\mathbb{1}^{1}$

The combination of in vitro multi-electrode arrays (MEAs) and the neuronal differentiation of stem cells offers the capability to study human neuronal networks from patient or engineered human cell lines. Here, we use MEA-based assays to probe synaptic function and network interactions of hiPSCderived neurons. Neuronal network behaviour first emerges at approximately 30 days of culture and is driven by glutamate neurotransmission. Over a further 30 days, inhibitory GABAergic signalling shapes network behaviour into a synchronous regular pattern of burst firing activity and low activity periods. Gene mutations in L-type voltage gated calcium channel subunit genes are strongly implicated as genetic risk factors for the development of schizophrenia and bipolar disorder. We find that, although basal neuronal firing rate is unaffected, there is a dose-dependent effect of L-type voltage gated calcium channel inhibitors on synchronous firing patterns of our hiPSC-derived neural networks. This demonstrates that MEA assays have sufficient sensitivity to detect changes in patterns of neuronal interaction that may arise from hypo-function of psychiatric risk genes. Our study highlights the utility of in vitro MEA based platforms for the study of hiPSC neural network activity and their potential use in novel compound screening.

Understanding how neurons interact both within local networks or as components of neural circuits is a key objective for the investigation of brain function throughout development and in neuropsychiatric disorders. Study of neural interactions in animals in vivo, in intact brain slices or in isolated primary neurons in culture has been a mainstay of neuroscience for more than a century. This has been augmented by human studies investigating functional (EEG and MEG) and structural (MRI) connectivity. However, the study of the development and function of human neural networks at the cellular level has been more difficult, largely due to the difficulty of combining cell culture methods and longitudinal electrophysiological experimentation. This is now changing with the opportunity to develop neurons derived from human induced pluripotent stem cells (hiPSC) and the advent of user-friendly multi-electrode array (MEA) systems. These offer the potential to generate small-scale human neural networks within a familiar context and investigate their development and function in vitro ${ }^{1}$.

Dissociated primary rodent neurons in vitro self-organise to form functional networks ${ }^{2-4}$, which spontaneously exhibit coordinated action potential bursts (synchronised bursts; SBs) that can be detected across spatially separated electrodes of an MEA ${ }^{5,6}$. This culture-wide, synchronised bursting behaviour correlates with an increased basal firing rate and emerges after an approximate two-week period of asynchronous firing. Recently, it has been shown that this synchronous behaviour may develop further to form a temporal pattern of regular low and high activity periods lasting for tens of seconds ${ }^{7}$, behaviour which is sensitive to pharmacological manipulation and in particular AMPA receptor activity.

MEA methods to study neuronal networks derived from hiPSC are less established. However, a number of MEA studies have shown that human stem cell-derived neurons develop spontaneous activity ${ }^{8}$, which increases during an extended period of development ${ }^{9-12}$. With prolonged incubation, the emergence of short SBs across multiple electrodes of the array has been observed ${ }^{10}$. These studies have established the use of MEAs for pharmacological profiling and toxicology testing ${ }^{8,10,13}$. However, it is currently not known whether such methods have the discriminatory sensitivity to investigate the effects of genetic risk in patient hiPSCs derived cultures, thereby offering a platform for drug development.

${ }^{1}$ Neuroscience and Mental Health Research Institute, Cardiff University, Cardiff, CF24 4HO, UK. ${ }^{2}$ Neuroscience, IMED Biotech Unit, AstraZeneca, 35 Gatehouse Dr, Waltham, MA, 02451, USA. ${ }^{3}$ Department of Neuroscience, Tufts University School of Medicine, Boston, MA, USA. Correspondence and requests for materials should be addressed to A.J.H. (email: harwoodaj@cardiff.ac.uk) 

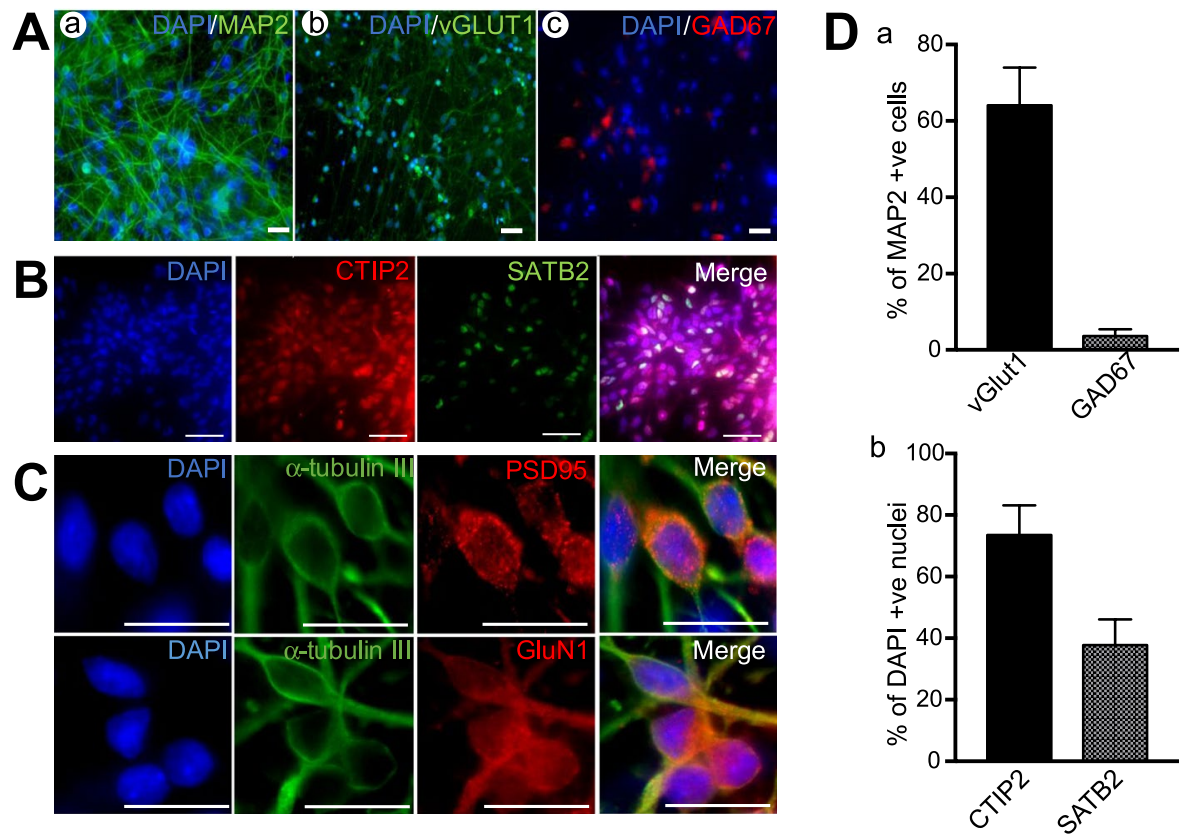

Figure 1. Morphology of hiPSC derived neurons. (A) Fluorescent images showing identity of differentiated neurons at 50 DPP. Neurons show expression of MAP2 (a), with a majority of these also expressing vGLUT1 (b) and a minority expressing GAD67 (c). (B) Layer marker expression in neurons at 50 DPP. (C) Expression of excitatory post-synaptic markers in neurons at $50 \mathrm{DPP}$. (D) Summary plots showing quantification of markers highlighting (a) neuronal fate (b) cortical layer markers. Scale bars in (A-C) $=50 \mu \mathrm{m}$. Plots in (D) show means \pm SD.

In this study, we demonstrate the use of MEA assays to probe synaptic function and functional neural networks in hiPSC-derived neuronal cultures. We first investigate the development of network behaviour in in vitro neuronal cultures on MEAs and use pharmacology to demonstrate a need for synaptic activity. We then use our hiPSC-based MEAs to probe the roles of L-type voltage-gated calcium channel activity in network behaviour. These channels have been implicated in several aspects of network function in vivo, including the synchronisation of calcium events ${ }^{14,15}$ and the regulation of hippocampal oscillatory activity ${ }^{16,17}$. Significantly, genetic variants in CACNA1C and CACNA1D (coding for the alpha- 1 subunits $\mathrm{Ca}_{\mathrm{v}} 1.2$ and $\mathrm{Ca}_{\mathrm{v}} 1.3$ respectively) are some of the most robust genetic risk alleles associated with the development of mental health disorders, including schizophrenia and bipolar disorder. By use of selective L-type channel inhibitors we demonstrate a specific modulation of SB pattern in hiPSC-derived neural networks without changes in basal neuronal activity. These results demonstrate the potential of hiPSC-based MEA assays to detect functional changes arising from genetic risk loci for psychiatric disorders.

\section{Results}

Creation of hiPSC-derived neuronal networks. Neurons were differentiated from hiPSC by a dual-SMAD monolayer protocol and re-plated on MEAs at 30DIV. MEA cultures were maintained in astrocyte conditioned medium (ACM) and in a $2 \% \mathrm{O}_{2}$ atmosphere to enhance neuronal maturation ${ }^{18,19}$. Parallel cell cultures to those on MEAs were examined by immunocytochemistry at 50 days post re-plating ( $50 \mathrm{DPP}$; corresponding to 50 days after cells were re-plated on MEAs or 80 days of total differentiation; Fig. 1). All imaged neurons expressed the neuron-specific microtubule associated protein MAP2, of which $64 \pm 9.0 \%$ co-expressed the vesicular glutamate transporter vGLUT1 and $3.6 \pm 1.2 \%$ of cells expressed the GABA synthesising enzyme GAD67 (Fig. 1A,D), indicating a mixed culture of predominately glutamatergic neurons with a small population of GABAergic cells. We also found that $73.5 \pm 9.7 \%$ of nuclei expressed CTIP2 with $37.7 \pm 8.4 \%$ also expressing SATB2 (Fig. 1B,D), suggesting a deeplayer cortical identity of most neurons primarily corresponding to layers 6 and 5a. Higher magnification images revealed that neurons also expressed the key excitatory synaptic scaffolding protein PSD95 and the obligatory glycine-binding NMDA receptor subunit, GluN1, suggesting the presence of excitatory synapses (Fig. 1C).

To confirm functional maturation of our hiPSC-derived neurons, we performed single-cell patch clamp experiments (Fig. 2A) to examine the basal physiological state of the neurons. Resting membrane potential $\left(\mathrm{V}_{\mathrm{m}}\right)$ and input resistance of neurons significantly reduced throughout differentiation $(-30.6 \pm 10.2 \mathrm{mV}$ to $-44.8 \pm 10.0 \mathrm{mV}$ and $1.26 \pm 0.66 \mathrm{G} \Omega$ to $0.92 \pm 0.41 \mathrm{G} \Omega$ respectively), indicating that neurons developed over time (Fig. 2B), although no change of the membrane time constant (tau) was observed. Patched neurons were classified according to their ability to fire induced and spontaneous action potentials (iAP and sAPs respectively; Fig. 2A,C). Cells were held at $-70 \mathrm{mV}$ via constant current injection and iAPs were elicited by injecting additional depolarising current steps. Neurons were subsequently coded based upon their voltage response (Fig. S1 and methods). At 50 DPP, $83.9 \%$ of neurons were classed as active, which included neurons showing single iAPs, 
A

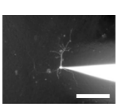

b

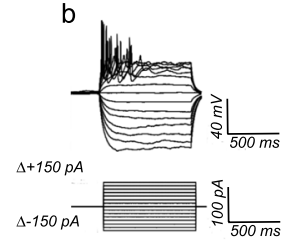

C

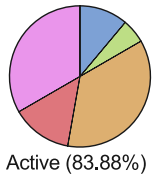

IAP classification

$\square$ None $(11.1 \%)$

$\square$ Attempted $(5.6 \%)$

$\square$ Single $(36.1 \%)$

Attempted trains $(13.9 \%)$

$\square$ Trains $(33.3 \%)$

\section{B}

\begin{tabular}{|c|c|c|c|}
\hline Property & 30DPP & 50DPP & t-test summaries \\
\hline \multicolumn{4}{|l|}{ Passive } \\
\hline$V_{m}(m V)$ & $-30.62 \pm 10.17$ & $-44.78 \pm 10.01$ & $\mathrm{t}_{49.28}=3.01 ; \boldsymbol{p}=\mathbf{0 . 0 0 4}$ \\
\hline Input resistance $(G \Omega)$ & $1.26 \pm 0.66$ & $0.92 \pm 0.41$ & $\mathrm{t}_{38.69}=2.69 ; \boldsymbol{p}=0.01$ \\
\hline Membrane time constant $(\tau)$ & $95.55 \pm 54.15$ & $74.44 \pm 37.35$ & $t_{37.29}=1.51 ; p=0.141$ \\
\hline \multicolumn{4}{|l|}{ Action potentials(induced) } \\
\hline Threshold $(m V)$ & $-23.67 \pm 4.53$ & $-26.32 \pm 7.15$ & $\mathrm{t}_{30.26}=1.18 ; p=0.247$ \\
\hline Amplitude ( $m V$ ) & $41.07 \pm 8.06$ & $45.28 \pm 8.88$ & $t_{30.96}=1.42 ; p=0.163$ \\
\hline Half width ( $m s$ ) & $6.61 \pm 2.02$ & $4.29 \pm 1.33$ & $\mathrm{t}_{20.91}=3.32 ; p=\mathbf{0 . 0 0 3 6}$ \\
\hline
\end{tabular}

C a
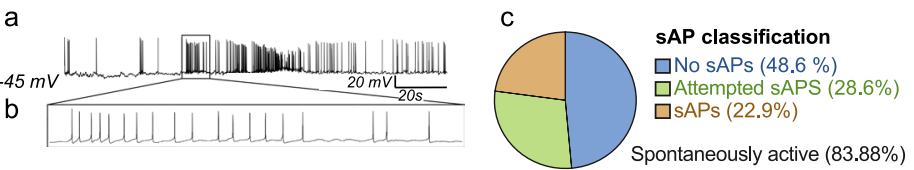

Figure 2. Intrinsic and action potential properties of hiPSC derived cortical neurons. (A) Induced action potential (iAP) formation in neurons. (a) Representative fluorescent image of a hiPSC derived neuron during single cell patching filled with Alexa Fluor 488 dye, highlighting pyramidal-like morphology. (b) Current injection protocol used for determination of passive and action potential properties of patched neurons. Cells were held at $-70 \mathrm{mV}$. (c) Classification of patched neurons based upon their induced action potential response. Full action potentials had an overshoot of $>0 \mathrm{mV}$; trains contained $\geq 2$ full action potentials. (B) Table summarising the intrinsic and action potential properties of patched iPS cell derived neurons. $\mathrm{N}=29$ (30 DPP), 36 (50 DPP). (C) Spontaneous action potentials (sAP) in hiPSC derived neurons. (a) Voltage trace showing presence of spontaneous activity in one patched cell. (b) Inset highlights period of higher-frequency firing. (c) Classification of patched cells based upon spontaneous action potential formation. Valid action potentials had overshoots of $>0 \mathrm{mV}$.

attempts at trains or full iAP trains (Fig. 2A). The AP threshold and average event amplitude of these iAPs was unchanged over development, however neurons did exhibit AP with faster rise times and shorter half-widths at 50 DPP compared to 30 DPP (Fig. 2B), highlighting the maturation of the cells over these 20 days. Finally, at 50 DPP, $22.9 \%$ of patched neurons exhibited sAPs, as determined by continuous recording of cells at their resting membrane potential ( $\mathrm{I}=0$; Fig. 2C).

Our single cell electrophysiology and imaging experiments indicated that our protocols yielded mature and functionally active neurons. To examine the development of networks in the cultures, differentiated hiPSC-derived neurons were plated as drop cultures onto MEAs possessing an $8 \times 8$ grid of substrate embedded electrodes, spaced $200 \mu \mathrm{m}$ apart. Spike detection from filtered raw voltage recording made from each electrode utilised the threshold-based method of QuianQuiroga and co-workers ${ }^{20}$ and following quality control spikes were timestamped for subsequent analysis. Spontaneous activity of cultures changed markedly over the 60 days of recorded development (Fig. 3A). Initial activity was very low with a mean firing rate of $0.065 \pm 0.021 \mathrm{~Hz}$ and no detectable spike bursts but increased to a firing rate of $0.75 \pm 0.21 \mathrm{~Hz}$ by $20 \mathrm{DPP}$ with $232 \pm 103$ bursts. The most substantial increase in activity occurred between 20 to 30 DPP with a 2-3-fold increase in spike rate and burst number, while after 30 DPP basal activity levels remain largely stable. These results are consistent with an increase in excitability previously seen throughout hiPSC-derived culture development ${ }^{8-11}$.

The emergence of synchronised bursting (SB) coincided with the switch to high neuronal activity levels seen at 30 DPP (Fig. 3A). This change in behaviour can be seen more clearly in raster and array-wide spike detection rate (ASDR) plots (Fig. 3B,C). At $30 \mathrm{DPP}$, SBs were short in length $(1.25 \pm 0.37 \mathrm{~s})$ with a firing rate of $107.60 \pm 87.06 \mathrm{~Hz}$ (Fig. 3D). Over the next 30 days, the number of SBs decreased, with a concomitant increase in both the length of the bursts and the interval between them $(17.07 \pm 8.34 \mathrm{~s}$ and $78.33 \pm 33.00 \mathrm{~s}$ respectively at $60 \mathrm{DPP})$. Although SB firing can be detected from $30 \mathrm{DPP}$, there was a clear shift in the pattern of array-wide activity from $50 \mathrm{DPP}$ into a regular pattern of more active periods (MAPs) and less active periods (LAPs) each lasting $>30$ seconds. Once networks have entered this state, the MAP/LAP pattern evolves further by increasing in MAP (SB) and LAP (SB interval) lengths (Fig. 3D). Finally, it should be noted that the changes in synchronised activity patterns occur with little change of overall basal neuronal activity (Fig. 3A).

Pharmacological profiling of hiPSC derived neuronal networks. To establish whether the extended SB firing patterns arise via synaptic activity, we probed our MEA cultures with pharmacological agents that alter glutamate/GABA signalling. At $20 \mathrm{DPP}$, when no SB activity is seen, neuronal cultures are unresponsive to AMPA, NMDA and $\mathrm{GABA}_{\mathrm{A}}$ receptor inhibition, but sensitive to $10 \mu \mathrm{M}$ GABA, which strongly attenuated spontaneous activity (Fig. S2). At the later 50 DPP stage when sustained SB firing has fully matured, inhibition of glutamate signalling by application of either the AMPA receptor antagonist CNQX or the NMDA receptor antagonist APV completely suppressed synchronised bursting (Fig. 4). In both cases, SB patterns rapidly re-establisherd after drug washout. Importantly, neither drug had a substantial effect on the basal rate of neuronal firing (Spike Rate) (Fig. 4Ca), indicating that both AMPA and NMDA receptor mediated neurotransmission is required for network synchrony, not single cell firing. 
A

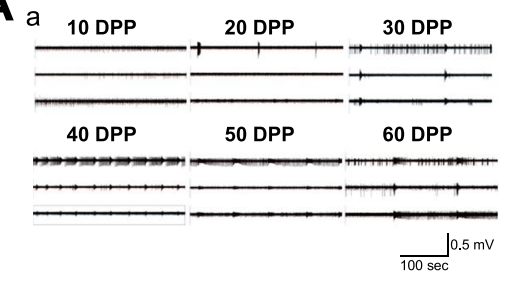

C

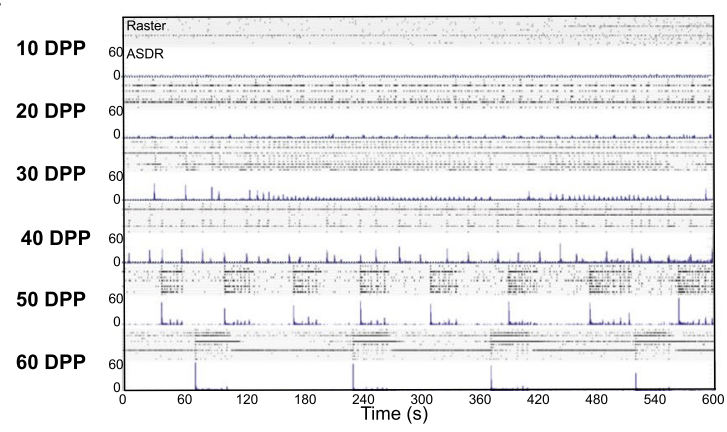

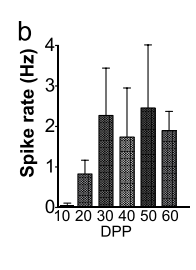

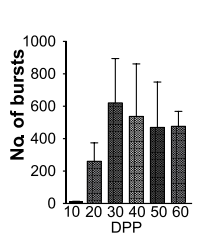

$D^{3}$

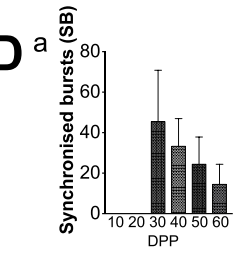

C

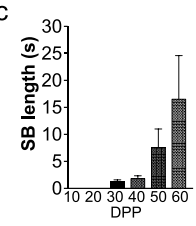

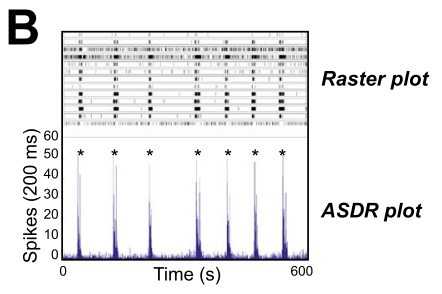

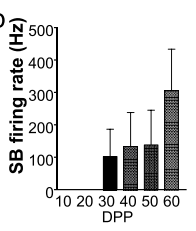

$d$

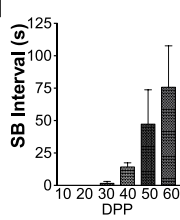

Figure 3. Changes in population activity of hiPSC derived neurons recorded with multi electrode arrays (MEAs). (A) (a) Representative voltage traces from three electrodes of the same MEA culture showing the changes in activity over a period of 60 days (DPP $=$ days post plating). (b) Basal excitability measured over culture development showing average spike rate and number of single unit bursts detected over 5 mins. (B) Conversion of time-stamp raster data to array wide spike detection rate (ASDR) plots, which form the basis of synchronised burst (SB) analyses. Plots are created by counting spikes in each $200 \mathrm{~ms}$ bin for each electrode, summing the counts for each bin and serially plotting the results. ASDR plots clearly show the occurrence of a culture-wide SB and allow the calculation of SB firing rates, lengths and intervals. (C) Development of array wide neuronal activity over 60 days of culture. For each time point, upper panel shows raster plot and lower panel shows ASDR plot. Vertical scale bars $=100$ spikes per $200 \mathrm{~ms}$ bin (D) Changes in synchronised burst (SB) activity over culture development showing (a) number of SBs detected over recording period (10 minutes); (b) SB firing rate - calculated as the number of spikes in individual SBs/length of that burst; (c) mean length of SBs and (d) mean interval between SBs. All summary data show means \pm SD.

Although the hiPSC differentiation protocol used here generates primarily cortical glutamatergic neurons, the resulting cultures also contain a small proportion on GABAergic neurons, which, in the nervous system, would be expected to exert an inhibitory effect on neural circuits and networks. To investigate the potential role of this inhibitory signalling on SB patterns, GABA or $\mathrm{GABA}_{\mathrm{A}}$ receptor antagonists were applied acutely to the MEA cultures. Exposure to $1 \mu \mathrm{M}$ GABA caused an almost complete, but reversible, loss of both basal and hence synchronised neuronal activity (Fig. 4), demonstrating that our hiPSC-derived glutamatergic neurons are GABA sensitive. In contrast, lowering the effect of endogenous GABAergic signalling within the culture by application of bicuculline, a GABA receptor blocker, significantly increased the number of $\mathrm{SBs}$ recorded $\left(\mathrm{t}_{10}=2.81\right.$; $\mathrm{p}<0.05)$. This was accompanied by a concomitant decrease in $\mathrm{SB}$ firing rate $\left(\mathrm{t}_{10}=1.91 ; \mathrm{p}<0.05\right)$ and in $\mathrm{SB}$ interval length (Fig. $4 \mathrm{C} ; \mathrm{t}_{10}=2.14 ; \mathrm{p}<0.05$ ). As a consequence, the general spike rate was not significantly altered, so that in effect reducing the effect of GABA increased the SB frequency without decreasing neuronal activity. These changes were reproduced when cultures were exposed to the non-competitive $\mathrm{GABA}_{\mathrm{A}}$ antagonist, picrotoxin (Fig. S3), strongly suggesting that this regulation is driven by the attenuation of GABAergic function.

Synchronised network activity in MEA cultures is regulated by L-type calcium channels. Calcium signalling via L-type voltage gated calcium (VGCCs) has been shown to be involved in the regulation of oscillatory behaviour in certain brain regions ${ }^{21,22}$. NMDA-mediated network signalling is thought to be regulated via calcium homeostasis mechanisms, partly through L-type $\mathrm{VGCCs}^{23,24}$. Moreover, recent genetic studies have strongly implicated L-type VGCCs in increased risk for a range of neuropsychiatric disorders, including schizophrenia, autism, depression and epilepsies; all pathologies with strong evidence for aberrant network signalling ${ }^{25,26}$. To investigate further the physiology of the neuronal cultures and to study the potential role of calcium in the underlying mechanism contributing to the network activity observed here, cells were exposed to the L-type VCCC blocker, Diltiazem.

Acute exposure of increasing doses of Diltiazem in 60 DPP day cultures had little effect on the basal firing properties of the neurons, specifically spike rate and single-unit bursts (Fig. 5Ba,Bb). However, application of Diltiazem induced a dose - dependent increase in the number of synchronised bursts (SB) and corresponding reduction in the interval between $S B s$, decreasing from $62.6 \pm 37.3 \mathrm{~s}$, to $39.5 \pm 11.4 \mathrm{~s}(1 \mu \mathrm{M})$, to $22.9 \pm 12.7 \mathrm{~s}$ $(2 \mu \mathrm{M})$ and finally to $20.5 \pm 9.4 \mathrm{~s}(5 \mu \mathrm{M}$; Fig. $5 \mathrm{Bc}, \mathrm{Bd})$. Dunnett's multiple comparison tests following one-way ANOVA $\left(F=6.004, \mathrm{R}^{2}=0.62, \mathrm{p}<0.01\right)$ revealed that the reduction in SB interval reached significance at 2 and $5 \mu \mathrm{M}$ (both $\mathrm{p}<0.05$ ). Average SB intervals returned to baseline following washes. The attenuation in SB intervals was replicated when cultures were exposed to the dihydropyridine-type L-type VGCC blocker Nifedipine 
A
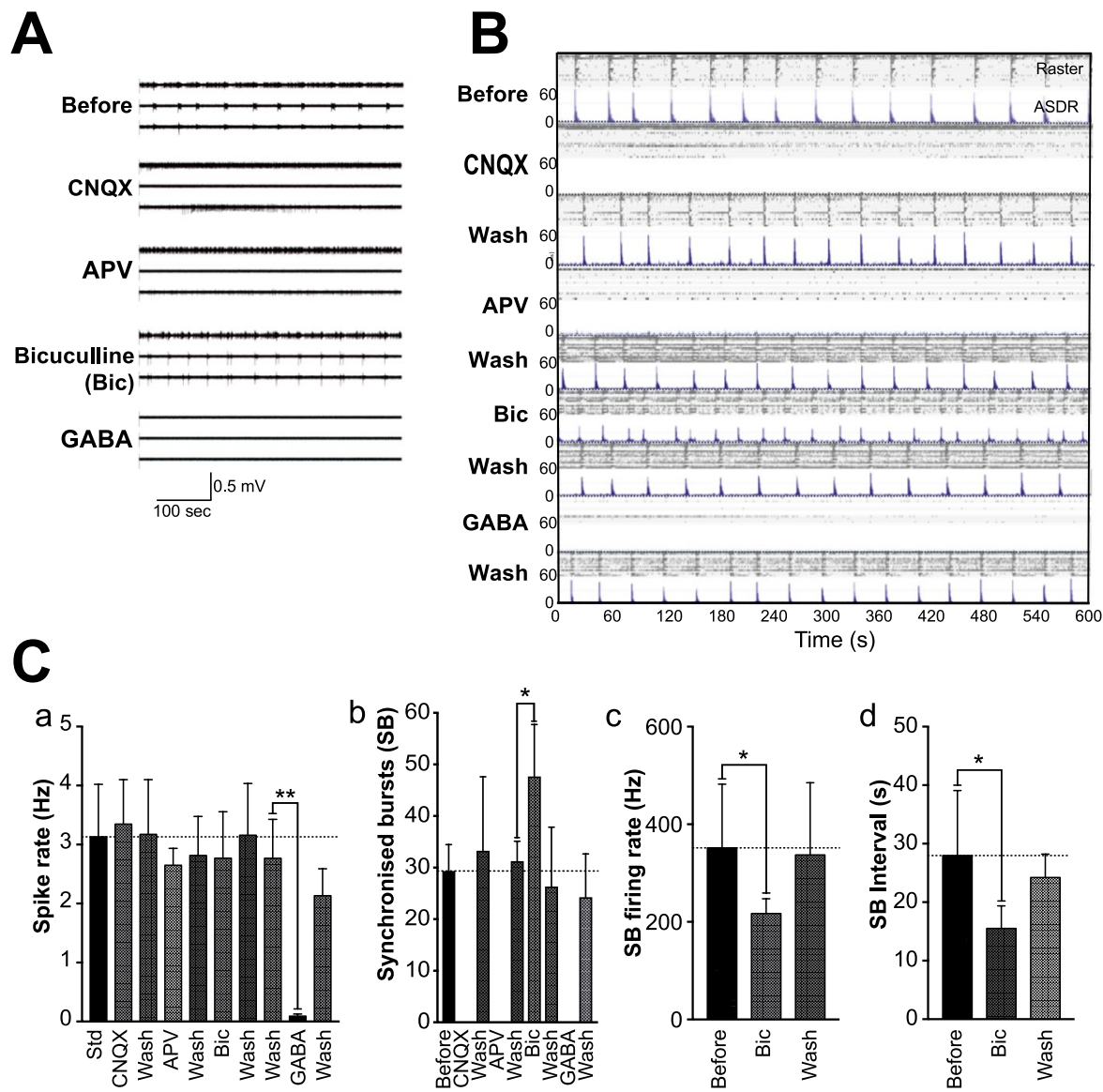

Figure 4. Pharmacological profiling of the network activity of hiPSC derived cortical neurons. (A) Filtered voltage traces of the same three electrodes recording the same MEA culture during acute exposure to NMDA, APV and $\mathrm{GABA}_{\mathrm{A}}$ receptor inhibitors. (B) Raster (upper panel) and ASDR (lower panel) plots of recordings of the same MEA showing the culture-wide response to acute exposure of CNQX $(50 \mu \mathrm{M}), \mathrm{APV}(50 \mu \mathrm{M})$, bicuculline $(\mathrm{Bic}, 10 \mu \mathrm{M})$ and GABA $(1 \mu \mathrm{M})$ at $50 \mathrm{DPP}$. Vertical scale bar $=200$ spikes per $200 \mathrm{~ms}$ bin. Established network activity in the cultures is dependent on both AMPA and NMDA receptor activity, while the coordinated behaviour is regulated by GABAergic tone. (C) Summary bar plots showing the response of MEA cultures to acute pharmacological treatment: (a) basal spike rate following all drug treatments and washes; (b) number of synchronised bursts (SB); (c) SB firing rate and (d) SB interval following bicuculline exposure. All plots show means $\pm \mathrm{SD}$. ${ }^{*} \mathrm{p}<0.05,{ }^{*} \mathrm{p}<0.01$ following paired t-tests. Number of arrays $=9$ at $50 \mathrm{DPP}$.

(Fig. 6A,B), strongly indicating that this observation was due to a reduction in L-type VGCC function. Both $C A C N A 1 C$ and CACNA1D genes, encoding the alpha 1 subunit of Cav1.2 and Cav1.3 respectively are expressed in neurons of these 60 DPP MEA cultures, however as Nifedipine has a 3-4 higher affinity to Cav1.2 than Cav1.3, inhibition of the former may be sufficient to alter network behaviour on MEAs.

To determine whether the effect on SB pattern was specific to the action of L-type VGCCs or Ca $\mathrm{a}_{\mathrm{v}}$ channel function more generally, the $\mathrm{Ca}_{\mathrm{v}} \mathrm{P} / \mathrm{Q}$ type blocker $\omega$-agotoxin $\mathrm{TK}$ and the $\mathrm{Ca}_{\mathrm{v}} \mathrm{T}$-type inhibitor ML218 were also applied acutely to cultures. Exposure of the cultures to both inhibitors had no effect on the basal firing rate and importantly on the SB interval (Fig. 6C,D). We did observe an apparent change in the shape profile of SBs after inhibiting $\mathrm{T}$ and $\mathrm{P} / \mathrm{Q}$ type channels, however this did not translate to a significant reduction in SB firing rates (Fig. 6Db) at these concentrations. These results, therefore, strongly suggest that the regulation of synchronised network activity observed in these hiPSC derived neuronal cultures is due to the action of L-type VGCCs.

\section{Discussion}

We have established conditions to monitor the development of human neuronal networks derived from hiPSCs as they self-assemble in vitro and transition from uncoordinated, spontaneously active cultures to complex oscillatory networks with close similarities to those previously reported with dissociated rodent primary neurons. We further show that this complex network behaviour can be targeted specifically by pharmacological agents without change to the underlying basal neuronal activity. To relate this process to those changes in network behaviour associated with mental health disorders, we demonstrated the specific effects of a pharmacological model of L-type voltage gated calcium channel hypo-function, a class of ion channels which have been strongly implicated as risk factors for psychiatric disorders. 
A

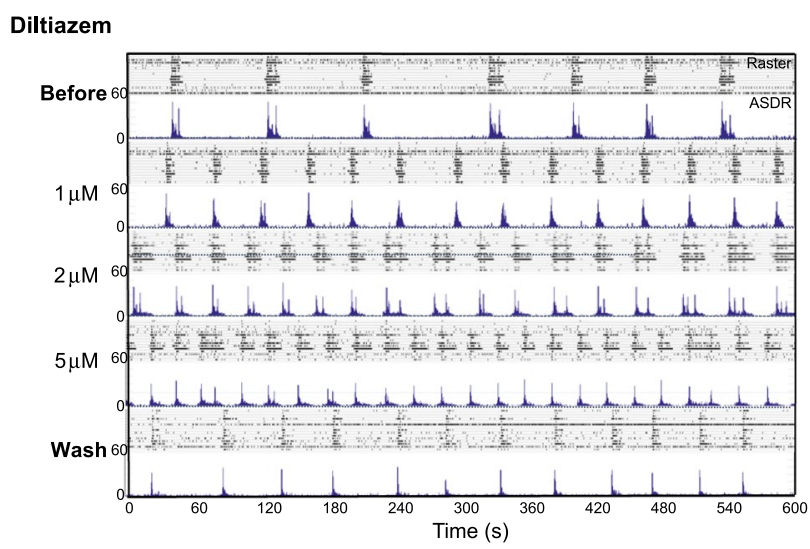

B a

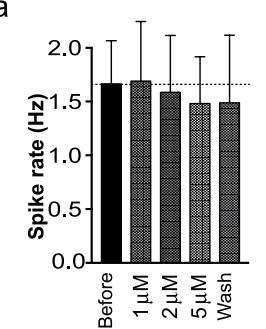

C

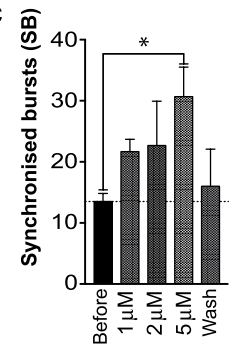

b

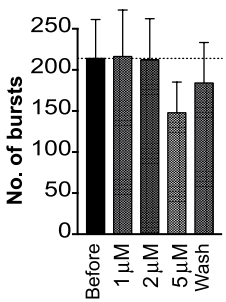

d

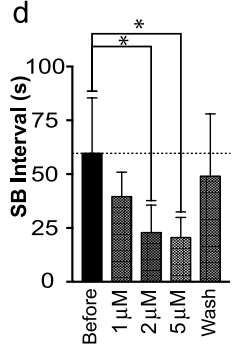

Figure 5. Network activity in hiPSC dervied neuronal cultures is regulated by L-type voltage gated calcium channels. (A) Raster (upper panels) and ASDR plots (lower panels) of a single neuronal MEA culture before, during and after acute exposure to increasing concentrations of the L-type calcium channel blocker Diltiazem at 60 DPP. Vertical scale bar $=200$ spikes per $200 \mathrm{~ms}$ bin. (B) Summary plots of basal and synchronised activity properties of MEA cultures in response to acute exposure to increasing doses of Diltiazem: (a) Basal average spike rate; (b) Average number of synchronised bursts; (c) Average culture-wide firing rate of SBs; (d) average interval between SBs. * $\mathrm{p}<0.05$ as determined from Dunnett's multiple comparisons tests following one way ANOVAs, where for Number of synchronised bursts $(\mathrm{Bb}) \mathrm{F}_{(7,21)}=3.324, \mathrm{R}^{2}=0.492 \& \mathrm{p}=0.0107$ and for $\mathrm{SB}$ interval $(\mathrm{Bd}) \mathrm{F}_{(7,21)}=6.004, \mathrm{R}^{2}=0.625 \& \mathrm{p}=0.0019$. All summary plots show means $\pm \mathrm{SD}$. Number of cultures $=7$.

Although the presence of synchronised array-wide activity has been reported previously in hiPSC-derived neuronal cultures ${ }^{10,27}$, this previously reported behaviour did not progress further than the short SB firing that we observed at $40 \mathrm{DPP}$ and did not evolve to the bi-stable period of oscillatory firing we report here. Indeed, this extended behaviour actually compares favourably to what has previously been described by several groups using similar MEA systems to study networks in dissociated rodent primary neurons ${ }^{3,5,28,29}$. In particular, the extended MAP/LAP oscillations we observed in hiPSC-derived neurons at 50 DPP matches to those reported in rodent studies at $\sim 20 \mathrm{DPP}^{7,29}$.

Pharmacological profiling of our cultures showed that glutamate/GABA signalling is the primary driver of the complex network behaviour observed. Whereas at initial stages spontaneous neuronal activity was insensitive to AMPA or NMDA receptor inhibition, we observed that synchronised network activity in these cultures was completely eliminated by inhibition of NMDA and AMPA receptor signalling. It has previously been suggested that blockade of NMDA but not AMPA receptors abolishes similar slow coordinated activity both in vivo and in in vitro rodent slices ${ }^{30-33}$. However, work with dissociated rodent neurons has also shown that the coordinated burst firing is indeed blocked by both AMPA and NMDA receptor inhibition ${ }^{2,34,35}$. It is therefore possible that the difference seen in dissociated neurons compared to 'intact' models is a function of the more random and heterogeneous nature of such cultures, which lack the intrinsic complexity, highly regulated developmental structure and region specific networking seen in slices and in vivo. Nonetheless, this adds to the utility of hiPSC-derived neuronal networks, as they can monitor both NMDA and AMPA receptor-based glutamate signalling.

Inhibition of $\mathrm{GABA}_{\mathrm{A}}$ receptor activity modifies the $\mathrm{SB}$ pattern in our mature networks, decreasing the interval between SB periods, a finding consistent with reports from both rodent dissociated primary cultures ${ }^{3,7,35}$ and hiPSC-derived neurons ${ }^{9,10}$. This indicates that although glutamate drives the complex network behaviour, GABA shapes it. The regulation of coordinated network firing by GABAergic interneurons is well documented, ranging from coordinating oscillatory activity across brain regions ${ }^{34,36,37}$, synchronised networks within structures (e.g. the hippocampus ${ }^{38,39}$ ) to regulation of small, localised networks ${ }^{40,41}$. Furthermore, GABAergic activity is thought to be highly important throughout development, where it has been implicated in the correct formation of networks and the regulation of synaptic plasticity ${ }^{42-44}$. Importantly, inappropriate GABAergic function leads to a non-physiological shift in the inhibitory/excitatory balance which is thought to underlie several neuropsychiatric disorders including schizophrenia, ASD and epilepsies.

By capturing the properties of glutamate/GABA interaction of neuronal networks, the MEA neuronal networks we report here offer a cell assay platform to study the effects of risk alleles associated with neuropsychiatric disorders. To pursue this further, we examined the effects of inhibition of the L-type VGCCs $\mathrm{Ca}_{\mathrm{v}} 1.2$ and $\mathrm{Ca}_{\mathrm{v}} 1.3$, the genes encoding the core subunits of which, CACNA1C and CACNA1D, are strongly associated with neuropsychiatric disorders. The L-type VGCC blockers of Diltiazem and Nifedipine caused a dose-dependent reduction of the SB interval with little change to the basal excitatory profile. This effect is specific to L-type channels and was not observed when T or P/Q channels were similarly blocked. 
A
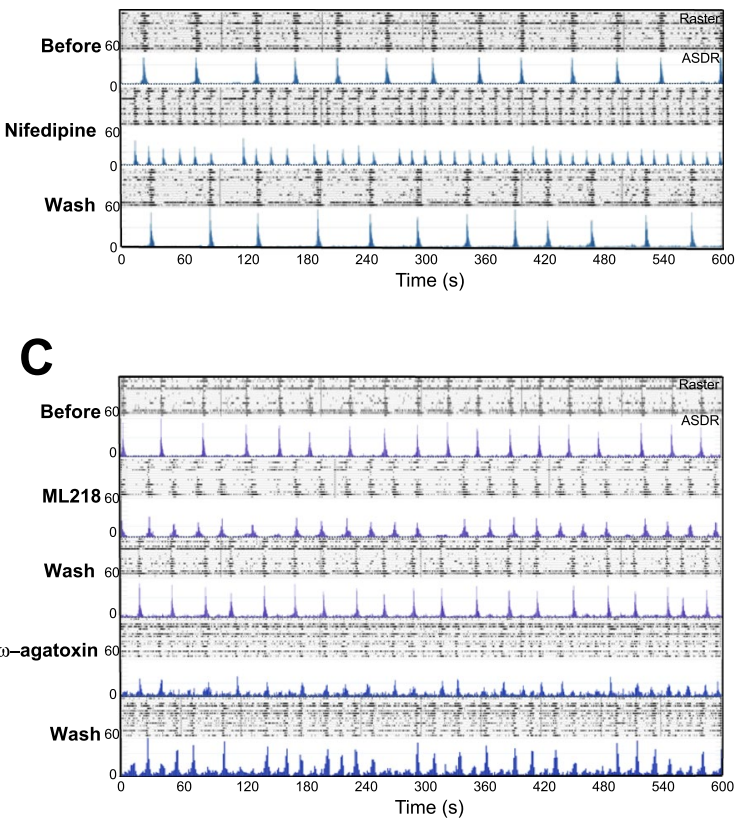

B

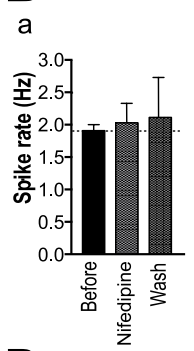

D

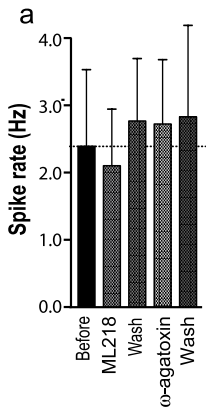

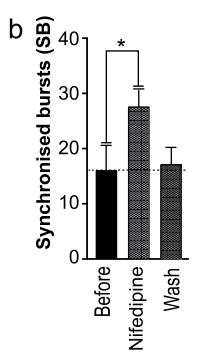

C

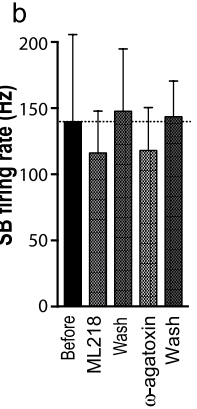

C

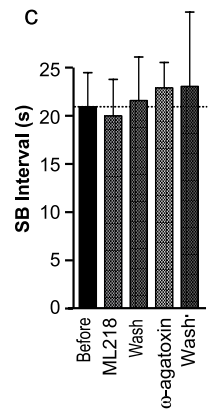

Figure 6. Effects on periodicity of synchronised burst firing in hiPSC derived neuronal cultures is specific to L-type and not T-type or P/Q type voltage-gated calcium channels. To determine whether the attenuation of intervals between synchronised bursts (SBs) was due to a generic reduction of general voltage gated calcium channel activity, neuronal cultures were exposed to the alternative L-type channel inhibitor Nifedipine, the T-type channel inhibitor ML218 and the P/Q-type blocker $\omega$-agatoxin TK. (A) Raster (upper panels) and ASDR (lower panels) plots showing the spontaneous activity of one MEA culture before, during and after exposure to drugs. (A) Raster and ASDR plots of a single 50 DPP MEA-culture before, during and after exposure to $1 \mu \mathrm{M}$ Nifedipine. (B) Summary plots showing average basal spike rate (a), number of synchronised bursts (b) and average interval between SBs (c). All plots show means $\pm S D$. $*$ in $(b)=p<0.05$ following paired t-test, Before vs Nifedipine, $\mathrm{t}_{3}=2.003$; in $(\mathrm{d})=\mathrm{p}<0.05$ following paired $\mathrm{t}$-test, Before vs Nifedipine, $\mathrm{t}_{3}=1.952$. (C) Raster (upper panels) and ASDR (lower panels) plots showing the spontaneous activity of one MEA culture before, during and after exposure to $1 \mu \mathrm{M}$ ML218 and $100 \mathrm{nM} \omega$-agatoxin. Drugs were washed out before further drug application. (D) Summary plots showing average (a) basal spike rate (a), SB firing rates (b) and average interval between $\mathrm{SBs}(\mathrm{c})$. All plots show means \pm SD from 7 cultures.

$\mathrm{Ca}_{\mathrm{v}} 1.2$ and $\mathrm{Ca}_{\mathrm{v}} 1.3$ alpha 1 subunits are expressed throughout the mammalian brain and are involved in wide array of calcium regulatory mechanisms ${ }^{45,46}$. In particular, they are strongly implicated in NMDA-dependant LTP/LTD, via regulation of local intracellular calcium concentrations ${ }^{47-49}$. This regulation of synaptic plasticity is thought to underlie the alterations to hippocampal - dependent learning seen with decreased or absent expression of L-type channels ${ }^{47,50,51}$. Furthermore, similar rodent KO studies have implicated L-type VGCCs in hippocampal-independent mechanisms of fear learning ${ }^{52-54}$, regulation of axon growth ${ }^{55}$, trafficking of AMPA receptor subunits ${ }^{56}$ and gene expression ${ }^{57,58}$.

A role for L-type VGCCs has also been described in a range of oscillatory activities in rodent neurons. Synchronised calcium transients have been shown to be mediated and controlled by L-type VGCCs in dissociated primary neurons $s^{22}$, while further studies have shown that these channels can regulate calcium oscillations in intact systems ${ }^{14,15,23}$. Importantly, it has also been shown that L-type VGCC currents can modulate the network response of neurons during physiological oscillatory behaviour in the hippocampus ${ }^{16,17,59}$ and during modelled epileptiform activity ${ }^{60,61}$. Involvement of L-type VGCCs in these systems is likely to be down to their role in the regulation of the post-burst after-hyperpolarisation (AHP), a period of hyper-polarisation which terminates high frequency firing ${ }^{62}$, where they have been shown to be a key mediator of the size and duration of the AHP ${ }^{62-64}$.

Our study presents for the first time the regulation of network activity in hiPSC derived neurons by modulating the function of L-type VGCCs. Importantly, this has implications for disease-modelling as mutations in the genes coding for L-type VGCCs represent some of the most strongly associated genetic risk factors for the development of mental-health disorders. Specifically, mutations in CACNA1C are amongst the most consistently detected genetic risk factors in both bipolar disorder and schizophrenia. Moreover, a gain-of function mutation in the pore forming region of $\mathrm{Ca}_{\mathrm{v}} 1.2$ is responsible for development of Timothy syndrome, a neurodevelopmental disorder characterised by cardiac arrhythmia, heart malformations and ASD ${ }^{65}$.

In our study, the action of Diltiazem and Nifedipine induced a similar effect on synchronised network behaviour (SB intervals) as that caused by inhibition of $\mathrm{GABA}_{\mathrm{A}}$ receptors. Is it possible that the two pharmacological pathways share an underlying mechanism? A potential scenario is that Diltiazem is acting via presynaptic L-type VGCCs on GABAergic interneurons nerve terminals. Indeed, L-type VGCCs have been shown be expressed in 
presynaptic terminals in the hippocampus ${ }^{66}$ and in interneurons ${ }^{67}$, while they have also been shown to interact with aspects of the exocytotic pathways and facilitate neurotransmitter release in certain neuronal populations ${ }^{68,69}$. Furthermore, calcium currents via L-type VGCCs have been shown to act through the MAPK ERK1/2 signalling pathway, which itself has been implicated in the regulation of vesicular exocytosis ${ }^{70,71}$. Importantly, this link has been observed directly as inhibition of the ERK1/2 pathway has been shown to increase neurotransmitter release via increased calcium influx via L-type VGCCs ${ }^{72}$. This therefore provides a potential mechanism by which L-type VGCCs may be acting to regulate the activity of GABAergic interneurons in these iPS cell derived cultures: synchronised network activity develops in cultures after maturation of functional synapses and is dependent on both AMPA and NMDA signalling; this activity is regulated by GABAergic innervation, inhibition of which attenuates the period between high frequency culture-wide firing; blocking L-type VGCCs mimics the effect of $\mathrm{GABA}_{\mathrm{A}}$ antagonism by reducing vesicular release at the interneuron-projection neuron synapse.

Our findings have shown that hiPSC derived neurons self-assemble in vitro to form complex, extended coordinated network firing. This has only previously been described in primary rodent cultures. This behaviour can be pharmacologically manipulated owing to the role of both intrinsic glutamatergic and GABAergic innervation and as such, serves as a physiologically relevant in vitro model of human neural development. Importantly, networks are sensitive to the specific attenuation of L-type VGCC function, thereby demonstrating the use of such models to monitor the function of disease relevant genetic hypo-function. The scalability of the developed MEA assays, together with increased knowledge about the genetics of mental health disorders and the increased availability of patient or engineered hiPSC lines should provide exciting opportunities for probing the function of human neural networks in disease relevant models and the potential development of novel therapeutics.

\section{Methods}

Cells culture and neuronal differentiation. All experiments were conducted using the IBJ4 cell line, a control iPS cell line derived from the BJ fibroblast cell line (ATCC; CRL-2522). iPS cells were differentiated into forebrain projection neurons using an adapted dual SMAD protocol ${ }^{73}$. Briefly, iPS cells were maintained on matrigel (Corning) and in mTeSR1 medium (StemCell Technologies). Prior to the start of differentiation, cells were passaged onto 12 well plated coated with growth factor reduced matrigel (Corning). Once cells were $>80 \%$ confluent (D0), medium was switched to N2B27 (2/3 DMEM/F12; 1/3 Neurobasal; B27-RA; N2; 1xPSG; 0.1 mM $\beta$-mecaptoethanol) $+100 \mathrm{nM} \mathrm{SB}$ and $100 \mathrm{nM} \mathrm{LDN} \mathrm{193189.} \mathrm{After} 8$ days SB and LDN were removed and cells were passaged onto fibronectin coated 12 well plates at a ratio of 2:3. After a further 8 days, cells were passaged onto PDL/laminin coated coverslips as single cells at a density of 400 cells $/ \mathrm{mm}^{2}$. Media was switched to N2B27 with B27+ retinoic acid, and $10 \mu \mathrm{M}$ DAPT for 7 days, after which medium was changed to astrocyte conditioned BrainPhys (Stem Cell Technologies ${ }^{74}$ ), supplemented with 1x B27, 1x PSG, $20 \mu \mathrm{M}$ ascorbic acid and $20 \mathrm{ng} / \mathrm{mL}$ BDNF, in which cells remained for the remainder of the cultures. For astrocyte conditioning of BrainPhys, $50 \mathrm{~mL}$ of medium was added to $60-80 \%$ confluent flasks of $<$ P5 Primary normal human astrocytes (Lonza), which were maintained previously in astrocyte medium (DMEM/F12 + 10\% FBS and 1x PSG). Medium was conditioned for 72 hours at $37^{\circ} \mathrm{C}$ in $20 \% \mathrm{O}_{2}$, after which conditioned medium was removed from flasks, sterilised using a $0.22 \mu \mathrm{m}$ syringe PVDF filter and stored at at $4{ }^{\circ} \mathrm{C}$ for use with 7 days or $-20^{\circ} \mathrm{C}$ for longer storage. Conditioned medium was used 1:1 with fresh BrainPhys.

Single cell electrophysiology. Patch clamping was performed on neurons using an electrophysiology rig comprising an Olympus BX51 WI upright microscope with 10x and 40x water immersion objectives; a Q-imaging Rolera Bolt CMOS camera; Luigs and Neumann Junior manipulators; Axon Instruments HS-2 Unity gain headstages; Axon Instruments Multiclamp 700B amplifier and Digidata 1550B; and a PC running Multiclamp commander. Cells were patched using borosilicate filament glass forged into pipettes using a Flaming/Brown puller (Sutter Instruments), resulting in resistances of 4-6 $\mathrm{M} \Omega$. All cells were patched at room temperature (around $22^{\circ} \mathrm{C}$ ) in a basic physiological extracellular fluid (ECF) comprising $142 \mathrm{mM} \mathrm{NaCl}$, $2.5 \mathrm{mM} \mathrm{KCL}, 2 \mathrm{mM} \mathrm{CaCl}_{2}, 1 \mathrm{mM} \mathrm{MgCl}, 10 \mathrm{mM}$ HEPES buffer and $30 \mathrm{mM}$ D-glucose; $\mathrm{pH}$ adjusted to 7.4 with $4 \mathrm{M} \mathrm{NaOH}$. The intracellular solution for pipettes consisted of $142 \mathrm{mM}$ potassium gluconate, $1 \mathrm{mM} \mathrm{CaCl}_{2}$, $2 \mathrm{mM} \mathrm{MgCl}_{2}, 10 \mathrm{mM}$ HEPES and $10 \mathrm{mM}$ EGTA; adjusted to $\mathrm{pH} 7.4$ with $\mathrm{KOH}$. The osmolality of internal solutions was adjusted to $290 \mathrm{mOsm}$, using a Vapour pressure osmometer (ELITech). After braking in to cells, resting membrane potentials were determined by taking the mean voltage from 2 minutes of gap free recording $(\mathrm{I}=0)$. Occurrence of induced action potentials (iAPs) was determined by holding cells at around $-70 \mathrm{mV}$ and injecting positive current steps $(\Delta 5-10 \mathrm{pA} ; 1$ second) until APs were seen. If no APs were seen when the membrane potential reached $-10 \mathrm{mV}$, it was assumed none would be seen at all. iAP categorisation of cells was based on visual assessment of traces. To be deemed a full AP, the overshoot had to be greater than $0 \mathrm{mV}$ otherwise they were deemed as 'attempted'. AP trains were determined as two or more full APs seen within the stimulus period (Fig. S1). Spontaneous action potentials were observed with gap free recording with cells at resting membrane potential $(\mathrm{I}=0)$. Compensation for fast and slow capacitive transients was applied after seal formation and series resistance (bridge balance) values were corrected after break in and were monitored throughout experiments. Recordings were taken using Clampex 10 (Molecular Devices, USA) and were subsequently analysed using Clampfit 10 (Molecular Devices, USA).

Multi electrode array culturing and recording. 60MEA200/30iR-Ti-gr MEAs were used throughout the study (Multi channel systems; MCS); planar MEAs with 60 titanium nitrate electrodes $(59+1$ internal reference) embedded within a silicon nitrate substrate. Each electrode had a diameter of $30 \mu \mathrm{m}$, with $200 \mu \mathrm{m}$ spacing between electrodes. MEAs were recorded using a MEA2100-HS2x60 headstage amplifier, attached into MSC-IFB-3.0 analogue/digital interface board. Cultures were recorded using the MCRack data acquisition software running on a high-performance PC. The recording head stage was isolated within a custom-made faraday cage. 
Coverslips of cells earmarked for arrays were re-plated onto MEAs at D40-45. Clean MEAs were stored at $4{ }^{\circ} \mathrm{C}$ in the dark, with the cultured area submerged in distilled water. Before cell plating, MEAs were first pre-treated with $1 \mathrm{ml} \mathrm{FBS}$ for 1 hour at $4^{\circ} \mathrm{C}$ in the dark, followed by washes with distilled water. Culture surfaces were then treated with $0.01 \%$ PEI (Sigma) and incubated for 1 hour at $37^{\circ} \mathrm{C}$, were then washed with distilled water and left to dry completely in a sterile cell culture hood 1 hour prior to re-plating. Neurons were dissociated from coverslips with Accutase (Thermo Fisher) and plated as high density drop cultures (50,000 cells/20 $\mu \mathrm{L}$, which equated to around 1800 cells $/ \mathrm{mm}^{2}$ ) containing $10 \mu \mathrm{g} / \mathrm{mL}$ laminin. After 1 hour, $1 \mathrm{~mL}$ of conditioned medium was then carefully flooded into each MEA and after a further 24 hours incubation, $1 \mathrm{ml}$ of fresh medium was added to each array. MEA cultures were maintained in 1:1 fresh/ACM BrainPhys and in an incubator with a $2 \% \mathrm{O}_{2}$ environment. A low oxygen incubator environment has been shown to increase the survival of neural precursors ${ }^{75}$ as well as promoting neuronal maturation ${ }^{18}$. All recordings were made at $37^{\circ} \mathrm{C}$.

All drugs used in this study were from Tocris (Bio-Techne Ltd.). For MEA drug experiments, stock concentrations were diluted as required in $1 \mathrm{~mL}$ 1:1 Brainphys:Brainphys ACM. Medium containing diluted drugs were added to MEAs and subsequently incubated for $10 \mathrm{mins}$ at $37^{\circ} \mathrm{C}$ in a standard $5 \% \mathrm{CO}_{2}$ incubator environment. After recordings, medium was removed from MEAs and cultures were washed 3 times with PBS. $1 \mathrm{ml}$ of fresh medium was then added and cultures were incubated for 5 minutes as before. This medium was then removed and replaced with fresh medium for 'Wash' recordings.

Raw MEA data was recorded at a sample rate of $25000 \mathrm{~Hz}$ and filtered online with a $200 \mathrm{~Hz}$ high pass and a $5000 \mathrm{~Hz}$ low pass filter (both $2^{\text {nd }}$ order butterworth). Recorded data was converted to ascii files for offline analysis using the MC Data tool (MCS). Offline analysis was achieved with custom scripts written in Matlab. Briefly, spikes were detected from filtered data using an automatic threshold-based method set at $-5.5 \times \hat{\sigma}$, where $\hat{\sigma}$ is an estimate of the noise of each electrode based upon the median absolute deviation $\left(\mathrm{MAD}^{20}\right)$. Spike timestamps were analysed to provide statistics on the general excitability of cultures. Network activity was analysed by creating array-wide spike detection rate (ASDR) plots with a bin width of $200 \mathrm{~ms}$. Synchronised bursts (SBs) were detected from ASDR plots by a 4-step process: 1; A 'start threshold' was determined as $\sim 10 \%$ of the maximum ASDR - this was varied to set the threshold just above baseline activity; 2. Data passing the start threshold was then required to pass a 'confirmation threshold' within 1 second - this was set as $70 \%$ of the maximum ASDR; 3 . The end of SBs was determined as there being 3 seconds of $<$ start threshold activity; 4 . SBs were finalised by confirming that they lasted at least 2 seconds. Average results for every measure were calculated as medians for each culture and data from single electrodes was excluded if it contained less than $5 \%$ of the spikes detected in the most active electrode (see Supplementary Information for full description of the analysis parameters).

Immunocytochemistry. Coverslips of cells for immunocytochemistry (ICC) were fixed as required using $4 \%$ paraformaldehyde solution (PFA; Sigma) and after washes were stored at at $4{ }^{\circ} \mathrm{C}$ in the dark until use. For staining, cells were first permeabilised using 0.1\% Triton X-100 (Sigma) diluted in DPBS, incubated at room temperature for 10 minutes and blocked by incubating with $1 \%$ bovine serum albumin (BSA) in PBST (DPBS $+0.1 \%$ Tween 20 ) for 30 mins at room temperature. Primary antibodies were diluted as required in PBST $+1 \%$ BSA incubated with cultures overnight at $4{ }^{\circ} \mathrm{C}$. Primary antibodies used in this study: mouse anti-MAP2 (Millipore, AB5622), chicken anti-TUJ1 (Neuromics, CH23005), mouse anti-GLUN1 (Antibodies Inc., 73-272), mouse anti-PSD95 (Antibodies Inc., 73-028), mouse anti-VGLUT1 (Antibodies Inc., 78-066), mouse anti-GAD67 (Millipore, MAB5406), rat anti-CTIP2 (Abcam, Ab18465) and mouse anti-SATB2 (Abcam, ab51602). The following day, cells were incubated with secondary antibodies diluted at 1:10000 in DPBS $+1 \%$ BSA for 1 hour at room temperature in the dark (Alexa Fluor 488, 594, and 647 - anti mouse, rabbit, chicken and rat as required; all Thermo Fisher) Cell nuclei were subsequently counter stained by incubating for 1 minute with $0.1 \mu \mathrm{g} / \mathrm{ml}$ DAPI (ThermoFisher \#62248) Coverslips were mounted onto slides using DAKO fluorescence mounting medium (Agilent) and imaged using a Leica DMI6000 inverted epifluorescence microscope. Images were processed with Fiji ${ }^{76}$.

Statistical analysis. All descriptive and comparative statistics were completed with Prism 6 (Graphpad. To determine the route of analysis (parametric or non-parametric), all data was first processed using histograms, q-q plots and normality tests (kurtosis and skew tests) in R. Where groups of data from the same experiment, presented with contrasting distributions, parametric tests were used as they are, in general, better equipped to cope with non-gaussian distributed data. Unless otherwise stated, all comparisons between pre- and post- treated cultures were done with paired t-tests and tests statistics, degrees of freedom and two-tailed p values are all reported. All summary plots of data show means + standard deviation. The numbers of arrays used for each experiment vary and are reported in individual figures. All results presented here were collected from at least three independent differentiations.

\section{References}

1. Spira, M. E. \& Hai, A. Multi-electrode array technologies for neuroscience and cardiology. Nat Nanotechnol 8, 83-94 (2013).

2. Maeda, E., Robinson, H. P. \& Kawana, A. The mechanisms of generation and propagation of synchronized bursting in developing networks of cortical neurons. J Neurosci 15, 6834-6845 (1995).

3. Chiappalone, M., Bove, M., Vato, A., Tedesco, M. \& Martinoia, S. Dissociated cortical networks show spontaneously correlated activity patterns during in vitro development. Brain Res 1093, 41-53 (2006).

4. Hales, C. M., Rolston, J. D. \& Potter, S. M. How to culture, record and stimulate neuronal networks on micro-electrode arrays (MEAs). J Vis Exp (2010).

5. Sun, J. J., Kilb, W. \& Luhmann, H. J. Self-organization of repetitive spike patterns in developing neuronal networks in vitro. Eur J Neurosci 32, 1289-1299 (2010).

6. Raichman, N. \& Ben-Jacob, E. Identifying repeating motifs in the activation of synchronized bursts in cultured neuronal networks. J Neurosci Methods 170, 96-110 (2008). 
7. Lu, C. et al. Micro-electrode array recordings reveal reductions in both excitation and inhibition in cultured cortical neuron networks lacking Shank3. Mol Psychiatry 21, 159-168 (2016).

8. Ylä-Outinen, L. et al. Human cell-based micro electrode array platform for studying neurotoxicity. Front Neuroeng 3 (2010).

9. Odawara, A., Saitoh, Y., Alhebshi, A. H., Gotoh, M. \& Suzuki, I. Long-term electrophysiological activity and pharmacological response of a human induced pluripotent stem cell-derived neuron and astrocyte co-culture. Biochem Biophys Res Commun 443, 1176-1181 (2014).

10. Odawara, A., Katoh, H., Matsuda, N. \& Suzuki, I. Physiological maturation and drug responses of human induced pluripotent stem cell-derived cortical neuronal networks in long-term culture. Sci Rep 6, 26181 (2016).

11. Amin, H. et al. Electrical Responses and Spontaneous Activity of Human iPS-Derived Neuronal Networks Characterized for 3-month Culture with 4096-Electrode Arrays. Front Neurosci 10, 121 (2016).

12. Paavilainen, T. et al. Effect of prolonged differentiation on functional maturation of human pluripotent stem cell-derived neuronal cultures. Stem Cell Res 27, 151-161 (2018).

13. Odawara, A., Matsuda, N., Ishibashi, Y., Yokoi, R. \& Suzuki, I. Toxicological evaluation of convulsant and anticonvulsant drugs in human induced pluripotent stem cell-derived cortical neuronal networks using an MEA system. Sci Rep 8, 10416 (2018).

14. Inglefield, J. R. \& Shafer, T. J. Polychlorinated biphenyl-stimulation of $\mathrm{Ca}(2+)$ oscillations in developing neocortical cells: a role for excitatory transmitters and L-type voltage-sensitive $\mathrm{Ca}(2+)$ channels. J Pharmacol Exp Ther 295, 105-113 (2000).

15. Bengtson, C. P., Kaiser, M., Obermayer, J. \& Bading, H. Calcium responses to synaptically activated bursts of action potentials and their synapse-independent replay in cultured networks of hippocampal neurons. Biochim Biophys Acta 1833, 1672-1679 (2013).

16. Hansen, A. K., Nedergaard, S. \& Andreasen, M. Intrinsic Ca2+-dependent theta oscillations in apical dendrites of hippocampal CA1 pyramidal cells in vitro. J Neurophysiol 112, 631-643 (2014).

17. Bukalo, O., Campanac, E., Hoffman, D. A. \& Fields, R. D. Synaptic plasticity by antidromic firing during hippocampal network oscillations. Proc Natl Acad Sci USA 110, 5175-5180 (2013).

18. Bilican, B. et al. Physiological normoxia and absence of EGF is required for the long-term propagation of anterior neural precursors from human pluripotent cells. PLoS One 9, e85932 (2014).

19. Rushton, D. J., Mattis, V. B., Svendsen, C. N., Allen, N. D. \& Kemp, P. J. Stimulation of GABA-induced Ca2+ influx enhances maturation of human induced pluripotent stem cell-derived neurons. PLoS One 8, e81031 (2013).

20. Quian Quiroga, R., Nadasdy, Z. \& Ben-Shaul, Y. Unsupervised Spike Detection and Sorting with Wavelets and Superparamagnetic Clustering. Neural Computation 16, 1661-1687 (2004).

21. He, J., Zhou, W., Zeng, S. \& Luo, Q. L-type calcium channels mediate synchronized spontaneous Ca2+spikes in cultured cortical networks. Conf Proc IEEE Eng Med Biol Soc 2, 1783-1785 (2005).

22. Przewlocki, R. et al. Opioid enhancement of calcium oscillations and burst events involving NMDA receptors and L-type calcium channels in cultured hippocampal neurons. J Neurosci 19, 9705-9715 (1999).

23. Wang, D., Grillner, S. \& Wallén, P. Calcium dynamics during NMDA-induced membrane potential oscillations in lamprey spinal neurons-contribution of L-type calcium channels (CaV1.3). J Physiol 591, 2509-2521 (2013).

24. Lee, K. Y. \& Chung, H. J. NMDA receptors and L-type voltage-gated $\mathrm{Ca}^{2+}$ channels mediate the expression of bidirectional homeostatic intrinsic plasticity in cultured hippocampal neurons. Neuroscience 277, 610-623 (2014).

25. Schizophrenia Working Group of the Psychiatric Genomics Consortium Biological insights from 108 schizophrenia-associated genetic loci. Nature 511, 421-427 (2014).

26. Zamponi, G. W., Striessnig, J., Koschak, A. \& Dolphin, A. C. The Physiology, Pathology, and Pharmacology of Voltage-Gated Calcium Channels and Their Future Therapeutic Potential. Pharmacol Rev 67, 821-870 (2015).

27. Matsuda, N. et al. Detection of synchronized burst firing in cultured human induced pluripotent stem cell-derived neurons using a 4-step method. Biochem Biophys Res Commun 497, 612-618 (2018).

28. Wagenaar, D. A., Pine, J. \& Potter, S. M. An extremely rich repertoire of bursting patterns during the development of cortical cultures. BMC Neurosci 7, 11 (2006).

29. Mok, S. Y., Nadasdy, Z., Lim, Y. M. \& Goh, S. Y. Ultra-slow oscillations in cortical networks in vitro. Neuroscience 206, 17-24 (2012).

30. Harsch, A. \& Robinson, H. P. Postsynaptic variability of firing in rat cortical neurons: the roles of input synchronization and synaptic NMDA receptor conductance. J Neurosci 20, 6181-6192 (2000).

31. Lazarewicz, M. T. et al. Ketamine modulates theta and gamma oscillations. J Cogn Neurosci 22, 1452-1464 (2010).

32. Hakami, T. et al. NMDA receptor hypofunction leads to generalized and persistent aberrant $\gamma$ oscillations independent of hyperlocomotion and the state of consciousness. PLoS One 4, e6755 (2009).

33. Molina, L. A., Skelin, I. \& Gruber, A. J. Acute NMDA receptor antagonism disrupts synchronization of action potential firing in rat prefrontal cortex. PLoS One 9, e85842 (2014).

34. Sanchez-Vives, M. V. \& McCormick, D. A. Cellular and network mechanisms of rhythmic recurrent activity in neocortex. Nat Neurosci 3, 1027-1034 (2000).

35. Chiappalone, M. et al. Networks of neurons coupled to microelectrode arrays: a neuronal sensory system for pharmacological applications. Biosens Bioelectron 18, 627-634 (2003).

36. Blatow, M. et al. A novel network of multipolar bursting interneurons generates theta frequency oscillations in neocortex. Neuron 38, 805-817 (2003).

37. Bruno, R. M. \& Sakmann, B. Cortex is driven by weak but synchronously active thalamocortical synapses. Science 312, 1622-1627 (2006).

38. Mann, E. O. \& Paulsen, O. Role of GABAergic inhibition in hippocampal network oscillations. Trends Neurosci 30, 343-349 (2007).

39. Bartos, M. et al. Fast synaptic inhibition promotes synchronized gamma oscillations in hippocampal interneuron networks. Proc Natl Acad Sci USA 99, 13222-13227 (2002).

40. Cobb, S. R., Buhl, E. H., Halasy, K., Paulsen, O. \& Somogyi, P. Synchronization of neuronal activity in hippocampus by individual GABAergic interneurons. Nature 378, 75-78 (1995).

41. Hu, H., Gan, J. \& Jonas, P. Fast-spiking, parvalbumin+ GABAergic interneurons: From cellular design to microcircuit function. Science 345, $1255263(2014)$

42. Higley, M. J. \& Contreras, D. Balanced excitation and inhibition determine spike timing during frequency adaptation. J Neurosci 26, 448-457 (2006)

43. Tremblay, R., Lee, S. \& Rudy, B. GABAergic Interneurons in the Neocortex: From Cellular Properties to Circuits. Neuron 91, 260-292 (2016).

44. Takada, N. et al. A developmental cell-type switch in cortical interneurons leads to a selective defect in cortical oscillations. Nat Commun 5, 5333 (2014).

45. Hell, J. W. et al. Identification and differential subcellular localization of the neuronal class C and class D L-type calcium channel alpha 1 subunits. The Journal of cell biology 123, 949-962 (1993).

46. Schlick, B., Flucher, B. E. \& Obermair, G. J. Voltage-activated calcium channel expression profiles in mouse brain and cultured hippocampal neurons. Neuroscience 167, 786-798 (2010). 
47. Moosmang, S. et al. Role of hippocampal Cav1. 2 Ca2+ channels in NMDA receptor-independent synaptic plasticity and spatial memory. Journal of Neuroscience 25, 9883-9892 (2005).

48. Malenka, R. C. \& Bear, M. F. LTP and LTD: an embarrassment of riches. Neuron 44, 5-21 (2004).

49. Zucker, R. S. Calcium-and activity-dependent synaptic plasticity. Current opinion in neurobiology 9, 305-313 (1999).

50. White, J. A. et al. Conditional forebrain deletion of the L-type calcium channel CaV1. 2 disrupts remote spatial memories in mice. Learning \& memory 15, 1-5 (2008).

51. Hofmann, F., Flockerzi, V., Kahl, S. \& Wegener, J. W. L-type CaV1.2 calcium channels: from in vitro findings to in vivo function. Physiol Rev 94, 303-326 (2014).

52. Cain, C. K., Blouin, A. M. \& Barad, M. L-type voltage-gated calcium channels are required for extinction, but not for acquisition or expression, of conditional fear in mice. Journal of Neuroscience 22, 9113-9121 (2002).

53. McKinney, B. C., Sze, W., White, J. A. \& Murphy, G. G. L-type voltage-gated calcium channels in conditioned fear: a genetic and pharmacological analysis. Learn Mem 15, 326-334 (2008).

54. Langwieser, N. et al. Homeostatic switch in hebbian plasticity and fear learning after sustained loss of Cav1.2 calcium channels. J Neurosci 30, 8367-8375 (2010).

55. Enes, J. et al. Electrical activity suppresses axon growth through $\mathrm{Ca}(\mathrm{v}) 1.2$ channels in adult primary sensory neurons. Curr Biol 20, 1154-1164 (2010).

56. Schierberl, K. et al. Cav1.2 L-type $\mathrm{Ca}^{2+}$ channels mediate cocaine-induced GluA1 trafficking in the nucleus accumbens, a long-term adaptation dependent on ventral tegmental area Ca(v)1.3 channels. J Neurosci 31, 13562-13575 (2011).

57. Wheeler, D. G. et al. $\mathrm{CaV} 1$ and $\mathrm{CaV} 2$ channels engage distinct modes of $\mathrm{Ca} 2+$ signaling to control CREB-dependent gene expression. Cell 149, 1112-1124 (2012).

58. Gomez-Ospina, N., Tsuruta, F., Barreto-Chang, O., Hu, L. \& Dolmetsch, R. The C terminus of the L-type voltage-gated calcium channel Ca V 1.2 encodes a transcription factor. Cell 127, 591-606 (2006).

59. Mohajerani, M. H., Sivakumaran, S., Zacchi, P., Aguilera, P. \& Cherubini, E. Correlated network activity enhances synaptic efficacy via BDNF and the ERK pathway at immature CA3 CA1 connections in the hippocampus. Proc Natl Acad Sci USA 104, 13176-13181 (2007).

60. Straub, H., Köhling, R., Frieler, A., Grigat, M. \& Speckmann, E. J. Contribution of L-type calcium channels to epileptiform activity in hippocampal and neocortical slices of guinea-pigs. Neuroscience 95, 63-72 (2000).

61. Empson, R. M. \& Jefferys, J. G. Ca(2+) entry through L-type $\mathrm{Ca}(2+)$ channels helps terminate epileptiform activity by activation of a Ca(2+) dependent afterhyperpolarisation in hippocampal CA3. Neuroscience 102, 297-306 (2001).

62. Lima, P. A. \& Marrion, N. V. Mechanisms underlying activation of the slow AHP in rat hippocampal neurons. Brain Res 1150, 74-82 (2007).

63. Shah, M. \& Haylett, D. G. Ca(2+) channels involved in the generation of the slow afterhyperpolarization in cultured rat hippocampal pyramidal neurons. J Neurophysiol 83, 2554-2561 (2000).

64. Gamelli, A. E., McKinney, B. C., White, J. A. \& Murphy, G. G. Deletion of the L-type calcium channel Ca(V) 1.3 but not Ca(V) 1.2 results in a diminished sAHP in mouse CA1 pyramidal neurons. Hippocampus 21, 133-141 (2011).

65. Splawski, I. et al. $\mathrm{Ca}(\mathrm{V}) 1.2$ calcium channel dysfunction causes a multisystem disorder including arrhythmia and autism. Cell 119, 19-31 (2004).

66. Tippens, A. L. et al. Ultrastructural evidence for pre-and postsynaptic localization of Cav1. 2 L-type Ca2+ channels in the rat hippocampus. Journal of Comparative Neurology 506, 569-583 (2008)

67. Westenbroek, R. E., Hoskins, L. \& Catterall, W. A. Localization of Ca2+ channel subtypes on rat spinal motor neurons, interneurons, and nerve terminals. J Neurosci 18, 6319-6330 (1998).

68. Wiser, O., Bennett, M. K. \& Atlas, D. Functional interaction of syntaxin and SNAP-25 with voltage-sensitive L-and N-type Ca2+ channels. The EMBO journal 15, 4100 (1996).

69. Wiser, O. et al. The voltage sensitive Lc-type Ca2+ channel is functionally coupled to the exocytotic machinery. Proc Natl Acad Sci USA 96, 248-253 (1999).

70. Ren, J. \& Guo, W. ERK1/2 regulate exocytosis through direct phosphorylation of the exocyst component Exo70. Dev Cell 22, 967-978 (2012)

71. Dolmetsch, R. E., Pajvani, U., Fife, K., Spotts, J. M. \& Greenberg, M. E. Signaling to the nucleus by an L-type calcium channelcalmodulin complex through the MAP kinase pathway. Science 294, 333-339 (2001).

72. Subramanian, J. \& Morozov, A. Erk1/2 inhibit synaptic vesicle exocytosis through L-type calcium channels. J Neurosci 31, 4755-4764 (2011).

73. Chambers, S. M. et al. Highly efficient neural conversion of human ES and iPS cells by dual inhibition of SMAD signaling. Nat Biotechnol 27, 275-280 (2009).

74. Bardy, C. et al. Neuronal medium that supports basic synaptic functions and activity of human neurons in vitro. Proc Natl Acad Sci USA 112, E2725-E2734 (2015).

75. Studer, L. et al. Enhanced proliferation, survival, and dopaminergic differentiation of CNS precursors in lowered oxygen. J Neurosci 20,7377-7383 (2000)

76. Schindelin, J. et al. Fiji: an open-source platform for biological-image analysis. Nat Methods 9, 676-682 (2012).

\section{Acknowledgements}

Thanks to Josh Chenoweth at the Lieber Institute for Brain Development for supplying the IBJ4 hiPS cell line. This work was supported by Welcome Trust Strategic Award (100202/Z/12/Z), The Waterloo Foundation 'Changing Minds' programme and AstraZeneca.

\section{Author Contributions}

Research was designed by A.J.H., W.P., J.H., N.B. and T.Z.D. All experiments were performed by W.P. The manuscript was written and reviewed by all authors.

\section{Additional Information}

Supplementary information accompanies this paper at https://doi.org/10.1038/s41598-019-50226-9.

Competing Interests: The authors declare no competing interests.

Publisher's note Springer Nature remains neutral with regard to jurisdictional claims in published maps and institutional affiliations. 
(c) (i) Open Access This article is licensed under a Creative Commons Attribution 4.0 International License, which permits use, sharing, adaptation, distribution and reproduction in any medium or format, as long as you give appropriate credit to the original author(s) and the source, provide a link to the Creative Commons license, and indicate if changes were made. The images or other third party material in this article are included in the article's Creative Commons license, unless indicated otherwise in a credit line to the material. If material is not included in the article's Creative Commons license and your intended use is not permitted by statutory regulation or exceeds the permitted use, you will need to obtain permission directly from the copyright holder. To view a copy of this license, visit http://creativecommons.org/licenses/by/4.0/.

(C) The Author(s) 2019 